\title{
Jolab: A free software to simulate light propagation in optical systems
}

\author{
Dylan M. Marques \\ Department of Medical Physics and \\ Biomedical Engineering \\ University College London \\ London, U.K. \\ dylan.marques.17@ucl.ac.uk
}

\author{
James A. Guggenheim \\ Department of Medical Physics and \\ Biomedical Engineering \\ University College London \\ London, U.K. \\ j.guggenheim@ucl.ac.uk
}

\author{
Peter R. T. Munro \\ Department of Medical Physics and \\ Biomedical Engineering \\ University College London \\ London, U.K. \\ p.munro@ucl.ac.uk
}

\begin{abstract}
An open source package for performing full-wave simulation of light propagation in optical systems is presented. The package includes pre-defined functions to model specific optical components. Optical systems are modelled simply by combining these functions. We outline its implementation and show examples of possible applications.
\end{abstract}

\section{Keywords—Computational optics, numerical modelling.}

\section{INTRODUCTION}

Optical engineering is important to various fields, from solar electricity production to the visualization of microscopic biological organisms. The design and optimization of optical systems is challenging since they are, in general, a combination of multiple, disparate, optical components. Some systems also possess parallel optical branches which are combined to perform interferometry. Optical models are very powerful for optimizing, understanding and creating optical setups. Well known proprietary, close source, software packages as VirtualLab Fusion (LightTrans GmbH, Germany), Code V (Synopsys, USA) and OpticsStudio (Zeemax, USA) are available at a cost. Alternatively, software packages as PROPER [1], Arroyo [2] and LightPipes [3] which are free but limited to specific applications

We present Jolab [4] a free and open source Julia package [5] that simulates full-wave and vectorial light propagation in general optical systems. The package models each optical component with the most appropriate theory, e.g. Fourier optics for lenses. Optical systems are simulated by modelling individual optical components sequentially, i.e. the light field output from one optical component is the input field on the next optical component.

\section{Simulating AN Optical SetuP}

Jolab is specifically designed to enable a very broad range or researchers, engineers and practitioners to simulate complex optical systems whilst requiring very little background modelling knowledge. This is made possible, in part, by the easy to follow design of the code. The functions available in Jolab are divided in three main categories: initialization of optical components, simulation of how light interacts with optical components and utility functions (e.g., data plotting).

To simulate an optical system the user must first define the optical components comprising the setup, then propagate the light field by the respective optical components in the respective order using the pre-built functions within Jolab. A
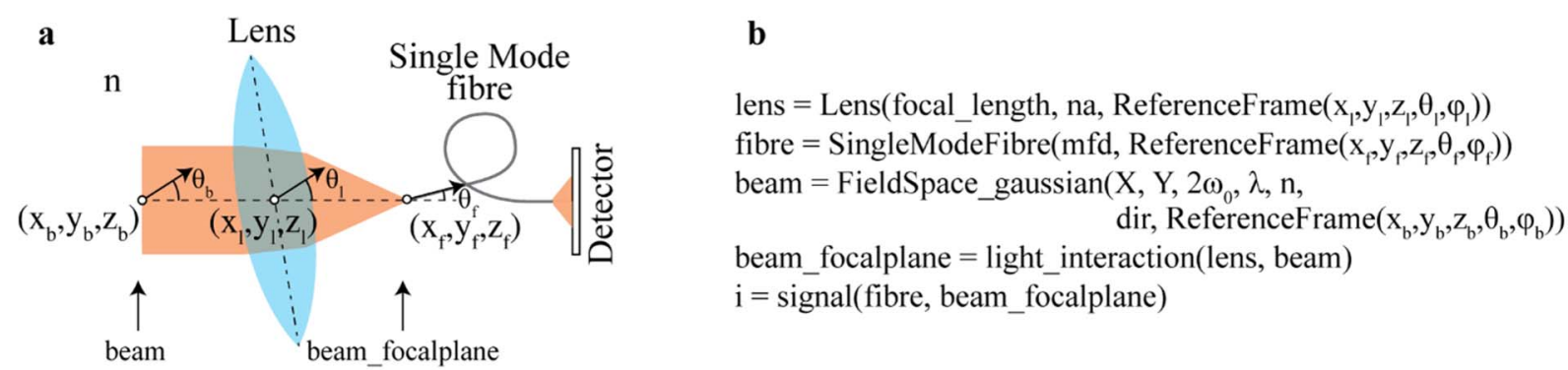

Figure 1-(a) An optical system for focussing a beam into a single mode fibre and measuring integrated intensity of coupled light. (b) Jolab code to simulate the optical system of (a). mfd is the Mode Field Diameter of the single mode fibre, $X$ and $Y$ are vectors of the spatial coordinates where the field will be evaluate, $2 \omega_{0}$ is the Gaussian spot size, $n$ is the refractive index of the media and dir is the direction in which the beam is propagating. 
simple example for calculating the light coupled into a single mode fibre when a Gaussian beam is focused on it is shown in Fig.1. More examples are available in [4].

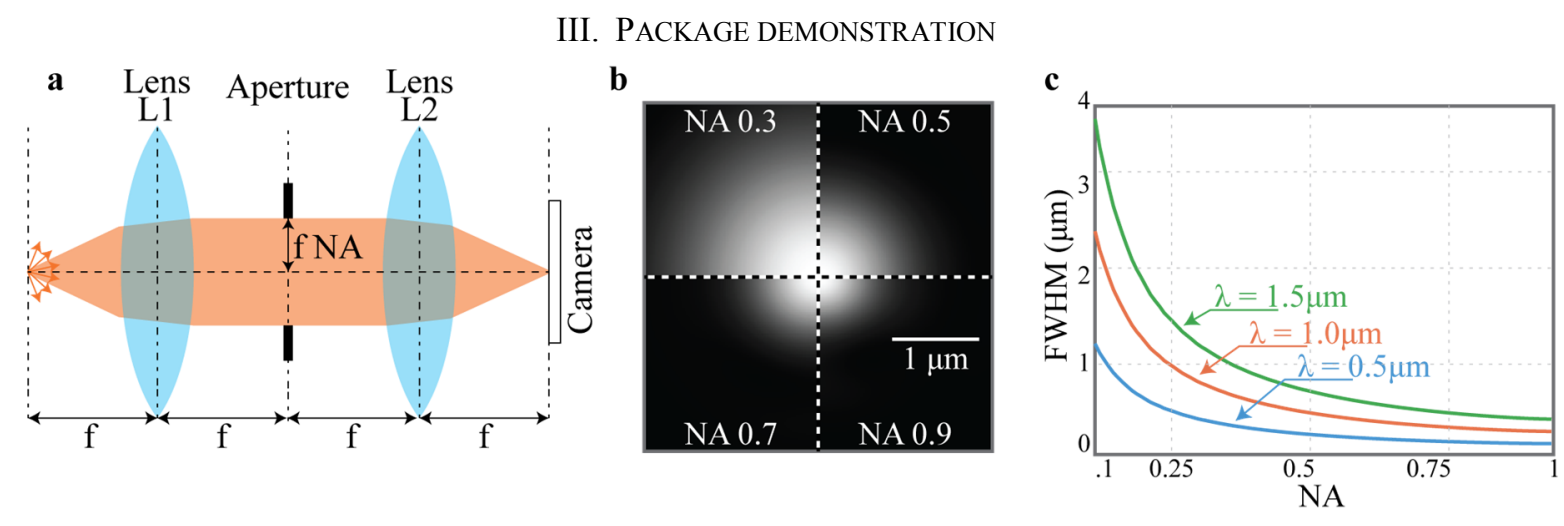

Figure 2 - (a) Optical system used to study the PSF as the NA of the $4 f$ system varies. (b) changes in the image acquired by the camera as the NA changes. (c) evolution of the FWHM of the PSF as a function of the NA for multiple wavelengths.

We demonstrate the application of Jolab in modelling optical systems. In the first example, the microscope of Fig.2a is simulated to study the impact of the numerical aperture (NA) on the resolution. A point source is assumed to be in the focal plane of the lens L1, and light propagate through the $4 \mathrm{f}$ system forming an image on the camera.

Fig.2b shows the microscope Point Spread Function (PSF) with 4 different NAs and, as the NA increases, the image resolution increase. The Full Width Half Maximum (FWHM) of the PSF was computed and plotted in Fig.2c to show the evolution of the system resolution with the NA and wavelength. As expected, the FWHM of the PSF is inverse proportional to the NA and proportional to the wavelength.

Further demonstrations will be made during the presentation including complex light field calculations, time dependent simulations and modelling alignment errors.

\section{CONCLUSION}

We have presented Jolab, a Julia package to simulate light propagation in optical systems using a very simple programmatic interface. Simulations are built up by setting the orientation and position of each optical component and light is propagated between and through components using pre-built functions.

We expect this package to be a very useful tool for designing, optimizing and analysing a range of optical systems. In future work, we will add further functions to Jolab including Mie theory, Finite-Difference Time-Domain (FDTD) and others. Our vision is for Jolab to become a standard in computational optics providing a free/open-source package for scientists and engineers to simulate optical setups such as Optical Coherence Tomography (OCT), ellipsometers, various types of microscopes, wavefront experiments, etc...

\section{REFERENCES}

[1] Krist, J. E. (2007, October). In Optical Modeling and Performance Predictions III (Vol. 6675, p. 66750P). SPIE.

[2] Britton, M. C. (2004, September). Modeling and Systems Engineering for Astronomy (Vol. 5497, pp. 290-300). SPIE.

[3] Bruegge, T. J., Rimmer, M. P., \& Targove, J. D. (1999, September). Optical Design and Analysis Software (Vol. 3780, pp. 14-22). SPIE

[4] Marques, D. M., Guggenheim, J. A, Munro P. R. T. (2020, April). Jolab. Retrieved from https://github.com/DylanMMarques/Jolab.j1

[5] Bezanson, J., Edelman, A., Karpinski, S., \& Shah, V. B. (2017). SIAM review, 59(1), 65-9 